\title{
Identification of reliable reference genes for gene expression studies in maternal reproductive tissues and fetal tissues of pregnant cows
}

\section{Lei Cheng}

Wuhan Academy of Agricutural Sciences

Jie Yu

Wuhan Academy of Agricultural Sciences

Xiuzhong Hu

Wuhan Academy of Agricultural Sciences

Min Xiang

Wuhan Academy of Agricultural Sciences

Yu Xia

Wuhan Academy of Agricultural Sciences

Bifei Tao

Wuhan Academy of Agricultural Sciences

Xiaoyong Du

Huazhong Agriculture University

Dingfa Wang

Wuhan Academy of Agricultural Sciences

Shuhong Zhao

Huazhong Agriculture University

Hongbo Chen ( $\square$ chen_hong_bo@126.com )

Wuhan Polytechnic University https://orcid.org/0000-0002-6051-3321

Research article

Keywords: Reference gene, RT-qPCR, Cow, Pregnancy

Posted Date: February 4th, 2020

DOI: https://doi.org/10.21203/rs.2.22639/v1

License: (1) (1) This work is licensed under a Creative Commons Attribution 4.0 International License.

Read Full License 


\section{Abstract}

Background: The relationship between the conceptus and the maternal uterine environment is crucial for the successful establishment and maintenance of pregnancy in cattle. Gene expression analysis of the conceptus and maternal reproductive tissues is a favorable method to assess the embryonic maternal interaction. The reliability of the commonly used method reverse transcription-quantitative polymerase chain reaction (RT-qPCR) depends on proper normalization to stable reference genes (RGs). The objective of this study was to determine the expression stability of ten potential RGs (SUZ12, CNOT11, ACTB, RPL 19, RPS9, GAPDH, TBP, HPRT1, SDHA and PPIA) in maternal reproductive tissues and fetal tissues, and to analyze the effect of $R G$ selection on the calculation of the relative expression of target genes.

Results: The expression stability of ten potential RGs was analyzed in eight different tissues (caruncular endometrium, intercaruncular endometrium, corpus luteum, ovary, oviduct, mammary gland, embryonic disc and trophoblast) from three pregnant dairy cows. Three programs-GeNorm, NormFinder and Bestkeeper-were used to identify the best RGs. According to all three programs, the most stable RG was CNOT11, whereas the least stable RGs were GAPDH and HPRT1. GeNorm analysis showed that a combination of five RGs (SDHA, PPIA, CNOT11, RPS9 and RPL19) was necessary for appropriate data normalization. However, NormFinder analysis indicated that the combination of CNOT11 and PPIA was the most suitable. When target genes were normalized to these RGs, the relative expression of the Radical Sadenosyl methionine domain containing 2 gene was not affected by the choice of RGs, whereas a large difference was observed in the expression profile of the Nuclear erythroid2-related factor 2 gene between the most stable RGs and least stable RGs.

Conclusions: The results indicate that careful selection of RGs is crucial under different conditions, especially for target genes with relatively small fold changes. Furthermore, the results provide useful information for the selection of RGs for evaluating genes affecting bovine reproduction.

\section{Background}

Reproductive performance substantially affects the profitability of dairy production. High rates of pregnancy loss, including both embryonic and fetal mortality during early gestation, are a key factor that decreases reproductive efficiency. The fertilization rate of dairy cattle is above $90 \%$, whereas the average calving rate is approximately $55 \%$; most pregnancy loss is thought to occur during the pregnancy recognition/pre-implantation period [1,2]. During the critical window, day 19 post insemination is a key time point for the initiation of implantation and the end of the maternal recognition of pregnancy [3]. The trophoblast cells are apposed to microvilli of the luminal epithelium of the endometrium on days 19-20 of pregnancy, and the trophoblast loosely communicates with the luminal epithelium on day $18[4,5]$. The ideal elongation of the embryo involves a complex interaction between the conceptus and the maternal system [6]. Notably, increased embryo loss during the pre-implantation period may be associated with alterations in conceptus-endometrium crosstalk. Investigating the potential interaction mechanisms between the conceptus and associated maternal tissues would not only improve understanding of 
pregnancy establishment and maintenance, but also aid in the development of management to decrease pregnancy loss.

The study of gene expression within the conceptus and maternal reproductive tissues is a favorable method to detect the embryonic maternal interaction in cattle. However, most previous studies have focused on either the conceptus or the maternal endometrium, but few studies have investigated the crosstalk between mother and conceptus, except for two pioneering studies using RNA-Seq [7] and microarray hybridization technologies [8]. Reverse transcription-quantitative polymerase chain reaction (RT-qPCR) is currently the most accurate and rapid method to validate high throughput transcriptomic results; it is also commonly used alone for gene expression measurement, even for genes with low transcription levels [9]. Because of the difficulty in determining the optimal normalization strategy to address the inherent variations in RT-qPCR studies, reference genes (RGs), also known as internal control genes or housekeeping genes, are commonly used in gene expression analysis [10]. An ideal RG should be consistently expressed in the samples regardless of the tissue, experimental condition or treatment. The choice of stably expressed genes for target gene normalization is crucial for accurate analysis of experimental results [11]. Unfortunately, no RG is generally appropriate in all contexts, and even the best RGs vary among different tissues [12]. Therefore, the selection of optimal RGs is an important prerequisite for RT-qPCR experiments.

Studies aimed at exploring the most suitable RGs in bovine species have been performed in specific tissues of the maternal reproductive system, such as the endometrium [13], endometrial explant [14], ovary [15], corpus luteum [16], myometrium [17] and mammary gland [18], as well as in embryos tissue under different experimental conditions $[19,20]$. However, RGs for expression analysis in both maternal reproductive tissues and fetal tissues have not been examined. Thus, the aim of this study was to assess the expression stability of ten candidate RGs (polycomb repressive complex 2 subunit (SUZ12), CCR4NOT transcription complex subunit 11 (CNOT11), actin beta (ACTB), Ribosomal protein L19 (RPL19), Ribosomal protein S9 (RPS9), Glyceraldehyde-3-phosphate dehydrogenase (GAPDH), TATA box binding protein (TBP), hypoxanthine phosphoribosyltransferase 1 (HPRT1), succinate dehydrogenase complex flavoprotein subunit $\mathrm{A}(S D H A)$ and peptidylprolyl isomerase $\mathrm{A}(P P I A))$ by using 24 samples representing maternal reproductive and fetal tissues (caruncular endometrium, intercaruncular endometrium, corpus luteum, ovary, oviduct, mammary gland, embryonic disc and trophoblast) from three pregnant dairy cows and to identify reliable RGs through three commonly used programs: GeNorm, Normfinder and Bestkeeper. Furthermore, two target genes, Nuclear erythroid2-related factor 2 (NRF2) and Radical $S$ adenosyl methionine domain containing 2 (RSAD2) were normalized to these RGs on the basis of expression stability, and the effect of RG selection on expression calculations for target genes involved in establishment and maintenance of pregnancy in bovine was also examined.

\section{Results}

\section{The amplification efficiencies and specificity of the candidate RGs}


To evaluate the expression stability of the ten selected genes under pregnancy conditions, we collected both maternal and fetal samples. In total, eight different types of tissues from three pregnant dairy cows were used for gene expression analysis with RT-qPCR. The RNA integrity and purity for each tissue were measured (Additional file 1: Fig. S1区Additional file 2: Table S1), and qualified samples were used for RTqPCR. The melting curve for each gene showed a single peak, thus indicating a single real time PCR product (date not shown). The amplification efficiencies of the primers varied from $85.0 \%$ to $100.4 \%$. The coefficient of determination varied between 0.917 and 0.999 (Table 1). The quantification cycle (Cq) values for all tissues ranged from 16.79 and 29.98 (Fig. 1). The Cq values for caruncular endometrium tissue ranged from 21.01 to 28.16 , whereas the $\mathrm{Cq}$ values for trophoblast tissue ranged from 16.79 to 27.92 (Fig. 1b, 1g).

\section{Gene expression stability analysis}

GeNorm software ranked the RGs according to their $M$ values, from the largest to smallest, and identified RPL 19 as the most stably expressed gene, followed by RPS9 and CNOT11, the commonly used reference gene GAPDH, and HPRT1, which was the least stably expressed gene (Fig. 2a). With the cut-off value of 0.15 , five or six of the best GeNorm RGs were suggested to normalize the gene expression results (Fig. 2b). Thus, SDHA, PPIA, CNOT11, RPS9 and RPL19 were selected as sufficient RGs by using GeNorm.

According to NormFinder, PPIA was the least variable gene, with a stability value of 0.394 , followed by CNOT11 (0.422) and RPL 19 (0.453) (Table 2). Similarly to the GeNorm analysis results, GAPDH and HPRT1 performed poorly as compared with all the other RGs, and had the highest stability values of 0.687 and 0.597 , respectively. Additionally, CNOT11 and PPIA were identified as the best RG combination, with a stability value of 0.264 .

Bestkeeper calculated an SD $>1$ for ACTB (1.47), GAPDH (1.84), SDHA (1.04), HPRT1 (1.05) and PPIA (1.13). The other five RGs showed SD $\leq 1$ and were used for further calculations: RPS9 (1.00), CNOT11 (0.81), TBP(0.90), RPL19 (0.79) and SUZ12 (0.71). Pair-wise correlation analysis was performed with Pearson correlation coefficients and $P$-values (Table 3$)$. The results showed a strong correlation $(r>0.8)$ for RPS9, CNOT11 and TBP as well as high significance levels $(P=0.001)$, and a medium correlation for $R P L 19$ and SUZ12, with high significance levels $(P=0.001$ and 0.004 , respectively). Therefore, $R P S 9$ and CNOT11 were identified as the best RGs according to Bestkeeper.

To combine the results of GeNorm, NormFinder and Bestkeeper programs, we calculated the average rank. According to Bestkeeper, ACTB, GAPDH, SDHA, HPRT1 and PPIA, with a high SD, were excluded from the calculation, and the average ranking of the remaining five candidates SUZ12, CNOT11, RPL 19, RPS9 and $T B P$ in the three programs was $6.67,2.33,2.67,2.33$ and 4.67 , respectively (Table 4). The results indicated that CNOT11 and RPS9 were the most stable RGs in the examined tissues, followed by RPL 19 .

\section{Effect of RG on target gene relative quantification}


To evaluate the effects of the above RG selections, we used RGs with various stability (Fig. 3) to calculate the relative expression of NRF2 and RSAD2 in both maternal reproductive tissues and fetal tissues. After normalization to the most stable gene (CNOT11) in all three programs, the optimum pair (CNOT11 and PPIA) of RGs according to NormFinder analysis, and the combination of the five best genes (SDHA, PPIA, CNOT11, RPS9 and RPL19) according to GeNorm analysis, the relative expression of NRF2 was lower in corpus luteum tissue and higher in the other tissues (Figure. 3a). However, the expression of NRF2 was lower in embryonic disc and trophoblast tissue as well as the corpus luteum when the least stable gene $(G A P D H)$ was used (Figure. $3 \mathrm{~b})$. When both the most stable RGs and the least stable RGs were used to normalize the expression of $R S A D 2$, the relative expression of $R S A D 2$ was low in embryonic disc and trophoblast tissues but higher in caruncular and intercaruncular endometrium tissues (Figure. 3c, d). The choice of control genes had no significant effect on the RSAD2 expression pattern, whereas it did affect the relative expression of NRF2 among all the tissues.

\section{Discussion}

The selection of suitable RGs for tissues of interest is critical for experimental design. Nevertheless, identifying universal RGs stably expressed in various types of tissues is difficult. Using RGs with varying expression may result in misinterpretation of gene expression data. Therefore, we evaluated the expression stability of ten candidate RGs in maternal reproductive tissues and fetal tissues of pregnant cows in the present study. Stability rankings obtained with the GeNorm, Normfinder and Bestkeeper algorithms revealed that $C N O T 11$ and RPS9 were the most stable genes among the ten candidates. They were also used as suitable RGs in other tissues. Previous RT-qPCR studies have shown that C2orf29, which is currently listed as CNOT11 in the NCBI database, has the highest stability in the bovine endometrium [13], corpus luteum [16] and myometrium [17], whereas RPS9 is the best RG in ovary [15] and mammary tissues [21]. The ranks of CNOT11 and RPS9 differed slightly among the three algorithms. CNOT11 was ranked second by both NormFinder and Bestkeeper, but third by GeNorm. RPS 9 was identified as the most stable gene by Bestkeeper, but second and fourth by GeNorm and NormFinder, respectively. Given the variability of the ranking, we recommend CNOT11 as the best RG in maternal reproductive tissues and fetal tissues of pregnant cows.

As mentioned above, the most stable RGs identified in RT-qPCR studies may differ depending on the statistical program used [22]. In our study, it should be emphasized that Normfinder selected PPIA as the best RG alone and as well as PPIA and CNOT11 as the best gene combination, whereas Bestkeeper determined a high SD for PPIA, indicating the least stability. Additionally, GeNorm showed moderate stability of PPIA. The different expression stability revealed by all of the three programs may have been because Normfinder considers the overall stability as well as any groups present in the tested samples, and it is not significantly influenced by co-regulation of candidate RGs [23]. Furthermore, it should be noted that the application of PPIA as RG was different in other tissues. PPIA has been found to be stable in the adipose tissue and mammary gland in goats [24], and it has been recommended as an RG for gene expression analysis of oocytes [25]. However, it is the most unstable gene in bovine adipose tissue, muscle, mammary gland and liver [18]. In agreement with our findings, PPIA expression was detected in 
many types of tissues. Our results highlight that validation of RGs in RT-qPCR studies is necessary through comparing the stability of potential genes according to the experiment, even if they have been used in previous studies.

GAPDH and HPRT1 were identified as the least stable genes as ranked by all the statistical programs. $G A P D H$ is one of the most frequently used RGs, but we do not recommend it because of its potential regulation in various physiological states. Its expression is changed by the presence of estrogens [26], and it has low expression stability in bovine ovaries [15], corpus luteum [16] and myometrium [17]. $H P R T 1$, a metabolism-related gene, has also been identified as the least stable gene in bovine ovaries [15] and myometrium [17]. Our results were in agreement with the findings of these studies: both GAPDH and HPRT1 showed the lowest stability among the candidates. However, Lesage-Padilla et al. have suggested that the combination of GAPDH, CNOT11 and SLC3OA6 is appropriate for accurate normalization in bovine endometrium [27]. Luchsinger et al. have reported that a combination of GAPDH, HMBS and EEF1A2 is optimal to normalize gene expression in bovine blastocysts [20]. In addition, HPRT1 together with other RGs has been used for normalization in cattle embryos [28]. The results suggest that the use of GAPDH or HPRT1 as the sole RG in maternal reproductive tissues and fetal tissues may not be feasible. Whether the two genes can be used in conjunction with other RGs requires further research.

In addition, GeNorm can indicate the optimal number of RGs that should be used in studies of gene expression (Fig. 2b). Our results indicated that V5/6 pairwise variation was below 0.15 , and five RGs were required for normalization of RT-qPCR data. It has been suggested that a minimum of three RGs be used between samples from the same tissue [29]. Cieslak et al. have proposed that a combination of four RGs is necessary for normalization in equine milk somatic cells [30]. Even more RGs are needed for analysis of oocyte gene expression from buffalos (seven) and cattle (nine) [25]. The samples detected in those studies involved one type of tissue or cell under different conditions. When the number of tissue types or species increases in GeNorm analysis, the number of RGs required for normalization may increase [18]. Saremi et al. have suggested that five RGs are required for data normalization in bovine adipose tissue and mammary glands [31], and the same number of RGs is also necessary for gene normalization in bovine adipose tissue, muscle, mammary glands and liver [18], in agreement with our results. In our study, the tested samples consisted of eight different tissues including maternal and fetal subgroups, and five RGs were needed to lower the $V$ and $M$ values and consequently decrease the technical standard error.

Finally, to confirm the effect of the selection of proper types and numbers of RGs in the gene expression studies, we assessed the expression of NRF2 and RSAD2 in maternal reproductive and fetal tissues of pregnant dairy cows by using four different groups of RGs with the most and least stability. We observed similar tendencies for NRF2 and RSAD2 after normalization to $C N O T 11$ (the best single gene according to the three programs); CNOT11 and PPIA (the best combination according to NormFinder); or SDHA, PPIA, CNOT11, RPS9 and RPL 19 (the best combination according to GeNorm). Interestingly, after normalization to the best single gene, CNOT11, and the worst single gene, GAPDH, there was a difference between NRF2 and $R S A D 2$. Although the relative expression of RSAD2 was low in embryonic disc and trophoblast tissues regardless of the RGs used, the relative expression of NRF2 was low in embryonic disc and 
trophoblast tissues only when GAPDH alone was used as the RG. RSAD2 is one of the 20 most abundant genes in endometrium tissues in early pregnancy in bovines, and its expression differs significantly between the endometrium and conceptus [7], thus possibly explaining why the relative expression of $R S A D 2$ was not affected by the choice of RGs. In agreement with our results, the expression of $P G R$ and OXTR among group comparisons has not been found to differ when RGs with different stability are used $[13,17]$. NRF2 plays an important role in protecting bovine mammary epithelial cells and bovine endometrial cells against oxidative damage [32,33]. However, the relative expression in maternal reproductive tissues and fetal tissues during early pregnancy is unknown. Our study is the first to investigate the relative expression of NRF2, and the results showed that NRF2 was low in the corpus luteum and higher in the other tissues after normalization to the most stable RG or combinations of RGs. NRF2 was low in the corpus luteum, embryonic disc and trophoblast after normalization to the least stable RG. The expression values of NRF2 in the embryonic disc and trophoblast were 1.80 and 2.16, respectively, after normalization to $C N O T 11$. The values were 0.39 and 0.30 , respectively, after normalization to GAPDH. The difference in the expression pattern of NRF2 confirmed that careful selection of the optimal RGs is important for the interpretation of the RT-qPCR results, especially for genes with low fold changes.

\section{Conclusions}

Under the conditions of the present study, the stability of the ten candidate RGs varied in the outcomes of the programs used. According to GeNorm, NormFinder and Bestkeeper analysis, CNOT11 is recommended as the best single RG. In contrast, GAPDH or HPRT1 should not be used as the sole RG for data normalization in maternal reproductive tissues and fetal tissues. The combination of five RGs (SDHA, PPIA, CNOT11, RPS9 and RPL 19) is necessary for appropriate data normalization according to GeNorm analysis, whereas the combination of CNOT11 and PPIA is most suitable according to NormFinder analysis. When the target genes were normalized to these RGs, a large difference was observed in the expression pattern of the NRF2 gene between the most stable and the least stable RGs, thus confirming that careful selection of the best RGs is critical for each experimental condition, especially when there are small differences in gene expression. The results obtained from this study provide valuable information for future gene expression analysis in bovines during pregnancy.

\section{Methods}

\section{Animals and treatments}

All animal procedures were performed according to protocols approved by The Hubei Province for Biological Studies Animal Care and Use Committee. Protocols were carried out in accordance with Hubei Provincial Regulation on Administration of Laboratory Animals (10/1/2005). License for all experimental animals was approved by Hubei Provincial Department of Science and Technology: SYXK(Hubei) 20140081. 
Six multiparous dairy cows were selected from a standard dairy farm of Wuhan Jinxu Animal Husbandry Science and Techbology Development Co. Ltd., China. The breeding system and fertility of those cows were normal, as confirmed by veterinary examination. Estrous synchronization and a superovulation program were implemented on the $9^{\text {th }}$ day of the estrus cycle. Pituitary follitropin (FSH; Ningbo Sansheng Pharmaceutical Co., Ltd., Ningbo, China) was injected intramuscularly at a dosage of 70 IU (a.m.), 70 IU (p.m.), $60 \mathrm{IU}, 60 \mathrm{IU}, 60 \mathrm{IU}, 60 \mathrm{IU}$ and $50 \mathrm{IU}, 50 \mathrm{IU}$ every day for 4 days (total dose, $480 \mathrm{IU}$ per cow). Cloprostenol sodium (Ningbo Sansheng Pharmaceutical Co., Ltd., $0.5 \mathrm{mg}$ ) was administered $60 \mathrm{~h}$ after the FSH treatment began, and Gonadorelin (Ningbo Sansheng Pharmaceutical Co., Ltd., $200 \mu \mathrm{g}$ ) was administered $108 \mathrm{~h}$ after the FSH treatment began. The animals were assessed for signs of estrus after the end of the injection, and five cows that came into standing estrus at similar times were used. All animals were kept in the same environment and inseminated with the semen from one bull.

\section{Tissue collection}

To ensure pregnancy success, we detected the steroid hormone progesterone (P4) in the peripheral blood once daily from day 0 to day 7 after estrus and from day 14 until slaughter. The pregnancy diagnosis on day 18 post insemination was confirmed by the detection of interferon stimulated genes (ISGs) expression in combination with P4 concentration (Additional file 3: Table S2). Three pregnant cows were head-only electrically stunned and subsequently slaughtered on day 19 at a local abattoir. The reproductive tract of each cow was collected within $30 \mathrm{~min}$ of slaughter and immediately placed on ice. The mammary gland tissues were sampled simultaneously. After the sample collections, an innocuity treatment for the dairy cows were performed following the standard operating rules of Hubei Provincial Regulation on Administration of Laboratory Animals. In the laboratory, the uterine horns ipsilateral to the ovary containing the corpus luteum were flushed with warm phosphate buffered solution (PBS). The collected embryos were observed under a stereomicroscope; for morphologically normal embryos $(n=24$; 8 recovered from each cow), the embryonic disc and the trophoblast were sampled and stored separately. The flushed uterine horns were opened longitudinally, and strips of endometrium (caruncular and intercaruncular) were carefully removed with curved scissors. The ovaries were cut longitudinally, the part of the zona parenchymatosa surrounding the corpus luteum was collected, and the corpus luteum tissues were also separated. The oviducts were separated from surrounding tissues, washed with sterile PBS, and transferred to Petri dishes. Subsequently, they were cut into three parts (isthmus, ampulla and infundibulum), and part of the ampulla was sampled. All tissues were cut to a maximum thickness of 0.5 $\mathrm{cm}$ in any one dimension, then immersed in five volumes of RNAlater (Sigma, St. Louis, MO) and stored for $24 \mathrm{~h}$ at $4^{\circ} \mathrm{C}$. Subsequently, these samples were transferred from RNAlater to RNA-free tubes and stored at $-80^{\circ} \mathrm{C}$ until RNA extraction.

\section{RNA extraction and cDNA synthesis}

The fetal tissues were washed with PBS three times before RNA extraction, and the maternal reproductive tissues were directly sectioned into smaller pieces and submerged in RNA isolation lysis solution. Total RNA was extracted with an RNAprep Pure Tissue kit (Tiangen Biochemical Technology Co. Ltd., Beijing, 
China). RNA concentration and purity were determined with a NanoDrop 2000 spectrophotometer (Thermo Electron Scientific Instruments LLC, Madison, WI USA), which measures the optical density (OD) at the absorption ratio $\mathrm{OD}_{260 \mathrm{~nm}}: \mathrm{OD}_{280 \mathrm{~nm}}\left(\mathrm{OD}_{260 \mathrm{~nm}}: \mathrm{OD}_{280 \mathrm{~nm}}\right.$ ranged from 1.9 to 2.1$)$; RNA integrity was detected through $1.2 \%$ agarose gel electrophoresis. After extraction, cDNA was synthesized from $500 \mathrm{ng}$ of total RNA with a RevertAid First-Strand cDNA Synthesis Kit (Thermo Scientific, Vilnius, Lithuania), the total reaction volume was $50 \mu$ l.

\section{Reverse transcription-quantitative polymerase chain reaction}

Ten RGs were selected to analyze gene expression stability (Table 1). The candidate RGs were selected because they had been used as RGs in bovines in previous studies $[14,15,17,19,28,34,35]$. The primers of the PPIA gene were designed with the online software Primer-BLAST (https://www.ncbi.nlm.nih.gov/tools/primer-blast/), and the other genes were referenced from previous studies $[15-18,35,36]$. The primers were synthesized by Tsingke Biotechnology Co. Ltd. (Wuhan, China). RT-qPCR, including the pre-experiment and formal experiment. In the pre-experiment, a standard curve for each primer pair was calculated. Briefly, cDNA from pooled samples was subjected to preamplification at the optimal annealing temperature. Standard curves were generated by RT-qPCR using five ten-fold serialdilutions of the preamplified products as templates. Amplification efficiencies (E) were determined for all candidate RGs through serial dilution with the formula $E=\left[10^{(1-s l o p e)}-1\right] \times 100$. The coefficient of determination indicated the linearity of the standard curve. The formal RT-qPCR was conducted in $20 \mu \mathrm{L}$ reactions containing $10 \mu \mathrm{L} 2 \times$ SYBR Green, $0.5 \mu \mathrm{L}(10 \mu \mathrm{mol} / \mathrm{L})$ forward and reverse primers, $0.5 \mu \mathrm{L} \mathrm{cDNA}$ template, DNase and RNase-free water (PCR-grade water). The cycling conditions were: $95^{\circ} \mathrm{C}$ for $60 \mathrm{~s}$; $95^{\circ} \mathrm{C}$ for $15 \mathrm{~s}$, annealing temperatures (Table 1) for $15 \mathrm{~s}$, and $72^{\circ} \mathrm{C}$ for $30 \mathrm{~s}$ for 40 cycles. For the melting curve and signal acquisition, the fluorescent quantitative PCR products were incubated for $5 \mathrm{~s}$ at each step with an increase in temperature of $0.5^{\circ} \mathrm{C}$ from $65^{\circ} \mathrm{C}$ to $95^{\circ} \mathrm{C}$ in each cycle. Each sample was repeated three times, and RT-qPCR was performed with a CFX Connect fluorescence quantitative PCR Detection System (Bio-Rad, Hercules, CA, USA).

\section{Statistical analysis}

Three algorithms-GeNorm [29], Normfinder [37] and Bestkeeper [10]-were used to evaluate the expression stability of the ten candidate RGs. GeNorm and Bestkeeper use raw $C q$ values, whereas in Normfinder analysis, the raw $\mathrm{Cq}$ values are transformed to relative quantities through the delta- $\mathrm{Ct}$ method. The GeNorm algorithm was implemented in QBASEPLUS software. The GeNorm algorithm determines the gene expression stability factor $(M)$, which was calculated as the average pairwise variation of one RG compared with all other RGs. M-values allow for ranking and sorting of the candidate RGs according to their expression stability. Lower values indicate the most stable genes. The lowest number of RGs needed for the optimal determination of gene expression can also be calculated as the GeNorm V value [25]. The Normfinder software estimates not only the variation of the candidate normalization genes but also the variation between the sample subgroup of the sample set. Therefore, all tissues included two groups of maternal reproductive tissues and fetal tissues. This program can also 
identify the pair of genes with the highest expression stability [26]. Bestkeeper, an Excel-based tool, calculates the standard deviation (SD) for each RG [10]. Pairwise correlation analysis was used to study the inter-gene relations of all RG combinations. The examined RGs with $S D>1$ were considered the least stably expressed. A Bestkeeper index was created from the stably expressed RGs $(S D<1)$ as a geometric mean of the $C q$ values from a maximum of ten RGs, which was then compared for each individual gene through pairwise correlation analysis, with each combination assigned a Pearson correlation coefficient value. The average rank was also calculated on the basis of the combined results of the GeNorm, NormFinder and Bestkeeper programs.

\section{Expression profile of target genes NRF2 and RSAD2}

NRF2 and RSAD2 were used as the target genes to determine the effects of RG selection on gene expression. CFX Connect software was used to perform expression profile analysis of target genes in maternal and fetal tissues of pregnant dairy cows. Expression of NRF2 and RSAD2 was determined with the most stable RGs and the least stable RGs, which were identified by all of the three programs. The reaction system and cycling conditions were the same as those for the RGs.

\section{Abbreviations}

RT-qPCR: reverse transcription - quantitative polymerase chain reaction; RGs: reference genes; SUZ12: polycomb repressive complex 2 subunit; CNOT11: CCR4-NOT transcription complex subunit 11; ACTB: actin beta; RPL19: Ribosomal protein L19; RPS9: Ribosomal protein S9; GAPDH: Glyceraldehyde-3phosphate dehydrogenase; TBP: TATA box binding protein; HPRT1: hypoxanthine phosphoribosyltransferase 1; SDHA: succinate dehydrogenase complex flavoprotein subunit A; PPIA: peptidylprolyl isomerase A; NRF2: Nuclear erythroid2-related factor 2; RSAD2: Radical S-adenosyl methionine domain containing 2; Cq: quantification cycle; P4: progesterone; ISGs: interferon stimulated genes; PBS: phosphate buffered solution; SD: standard deviation.

\section{Declarations}

\section{Ethics approval and consent to participate}

All animal procedures were performed according to protocols approved by The Hubei Province for Biological Studies Animal Care and Use Committee. Protocols were carried out in accordance with Hubei Provincial Regulation on Administration of Laboratory Animals (10/1/2005). License for all experimental animals was approved by Hubei Provincial Department of Science and Technology: SYXK(Hubei) 20140081.

\section{Consent for publication}

Not applicable 
Availability of data and material

All data generated or analyzed during this study are included in this published article.

\section{Competing interests}

The authors declare that they have no competing interests.

\section{Funding}

The design of the study and the implementation of the experiment were supported by the International Science and Technology Cooperation Programs of the Wuhan Science and Technology Bureau (2016030409010229). The collection and analysis of the data were supported by IAEA CRP project (CRPD3-10-28). The interpretation of the data and the writing of the manuscript were supported by the International Science and Technology Cooperation Programs of the Wuhan Science and Technology Bureau (2017030209010261).

\section{Authors' contributions}

LC and SHZ designed the study; LC wrote the paper; LC, JY, MX, YX, BFT and DFW carried out the experiments; $\mathrm{XZH}$ and XYD participated in data collection and analysis; $\mathrm{SHZ}$ and $\mathrm{HBC}$ conducted the study and prepared the manuscript. All authors read and approved the final manuscript.

\section{Acknowledgements}

The authors wish to thank Prof. Ke-Mei Peng (Huazhong Agricultural University) for help with morphological identification of the sampled tissues.

\section{References}

1. Diskin MG, Murphy JJ, Sreenan Embryo survival in dairy cows managed under pastoral conditions. Anim. Reprod. Sci. 2006; 96: 297-311.

2. Wiltbank MC, Baez GM, Garcia-Guerra A, Toledo MZ, Monteiro PL, Melo LF, Ochoa JC, Santos JE, Sartori R. Pivotal periods for pregnancy loss during the first trimester of gestation in lactating dairy cows. 2016; 86: 239-253.

3. Morris D, Diskin M. Effect of progesterone on embryosurvival. Animal. 2008; 2: 1112-1119.

4. King GJ, Atkinson BA, Robertson Development of the intercaruncular areas during early gestation and establishment of the bovine placenta. J. Reprod. Fertil. 1981; 61: 469-474.

5. Guillomot Cellular interactions during implantation in domestic ruminants. J. Reprod. Fertil. Suppl. 1995; 49: 39-51.

6. Hue I, Degrelle SA, Turenne Conceptus elongation in cattle: genes, models and questions. Anim. Reprod. Sci. 2012; 134: 19-28. 
7. Mamo S, Mehta JP, Forde N, McGettigan P, Lonergan P. Conceptus-endometrium crosstalk during maternal recognition of pregnancy in cattle. Reprod. 2012; 87: 6, 1-9.

8. Moore SG, McCabe MS, Green JC, Newsom EM, Lucy MC. The transcriptome of the endometrium and placenta is associated with pregnancy development but not lactation status in dairy cows. Reprod. 2017; 97: 18-31.

9. Bustin SA, Mueller Real-time reverse transcription PCR (qRT-PCR) and its potential use in clinical diagnosis. Clin. Sci. 2005; 109: 365-379.

10. Pfaffl MW, Tichopad A, Prgomet C, Neuvians Determination of stable housekeeping genes, differentially regulated target genes and sample integrity: BestKeeper-Excel-based tool using pairwise correlations. Biotechnol. Lett. 2004; 26: 509-515.

11. Janovick-Guretzky NA, Dann HM, Carlson DB, Murphy MR, Loor JJ, Drackley JK. Housekeeping gene expression in bovine liver is affected by physiological state, feed intake, and dietary treatment. Dairy. Sci. 2007; 90: 2246-2252.

12. Hruz T, Wyss M, Docquier M, Pfaffl MW, Masanetz S, Borghi L, Verbrugghe P, Kalaydjieva L, Bleuler S, Laule O, Descombes P, Gruissem W, Zimmermann P. RefGenes: identification of reliable and condition specific reference genes for RT-qPCR data normalization. BMC Genomics. 2011; 12: 156.

13. Walker CG, Meier S, Mitchell MD, Roche JR, Littlejohn Evaluation of real-time PCR endogenous control genes for analysis of gene expression in bovine endometrium. BMC Molecular Biology. 2009; 10: 100 .

14. Mathew DJ, Sánchez JM, Passaro C, Charpigny G, Behura SK, Spencer TE, Lonergan P. Interferon taudependent and independent effects of the bovine conceptus on the endometrial transcriptome. Reprod. 2019; 100: 365-380.

15. Schoen K, Plendl J, Gabler C, Kaessmeyer S. Identification of stably expressed reference genes for RTqPCR data normalizationin defined localizations of cyclic bovine ovaries. Histol. Embryol. 2015; 4: 200-11.

16. Rekawiecki R, Rutkowska J, Kotwica J. Identification of optimal housekeeping genes for examination ofgene expression in bovine corpus luteum. Biol. 2012; 12: 362-7.

17. Rekawiecki R, Kowalik MK, Kotwica J. Validation of housekeeping genes for studying differential geneexpression in the bovine myometrium. Vet. Hung. 2013; 61: 505-16.

18. Bonnet M, Bernard L, Bes S, Leroux C. Selection of reference genes for quantitative real-time PCR normalisation in adipose tissue, muscle, liver and mammary gland from ruminants. 2013; 7: 134453.

19. Goossens K, Van Poucke M, Van Soom A, Vandesompele J, Van Zeveren A, Peelman LJ. Selection of reference genes for quantitative real-time PCR in bovine preimplantation embryos. BMC Dev. Biol. 2005; 5: 27.

20. Luchsinger C, Arias ME, Vargas T, Paredes M, Sánchez R, Felmer R. Stability of reference genes for normalization of reverse transcription quantitative real-time PCR (RT-qPCR) data in bovine blastocysts produced by IVF, ICSI and SCNT. 2014; 22: 505-12. 
21. Bionaz M, Loor JJ. Identification of reference genes for quantitative real-time PCR in the bovine mammary gland during the lactation cycle. Genomics. 2007; 29: 312-9.

22. Kozera B, Rapacz Reference genes in real-time PCR. J. Appl. Genet. 2013; 54: 391- 406.

23. Beekman L, Tohver T, Dardari R, Léguillette R. Evaluation of suitable reference genes for gene expression studies in bronchoalveolar lavage cells from horses with inflammatory airway disease. BMC Mol. Biol. 2011; 12:

24. Bonnet M, Delavaud C, Bernard L, Rouel J, Chilliard Sunflower-seed oil, rapidly-degradable starch, and adiposity up-regulate leptin gene expression in lactating goats. Domest. Anim. Endocrinol. 2009; 37: 93-103.

25. Macabelli CH, Ferreira RM, Gimenes LU, de Carvalho NA, Soares JG, Ayres H, Ferraz ML, Watanabe YF, Watanabe OY, Sangalli JR, Smith LC, Baruselli PS, Meirelles FV, Chiaratti MR. Reference Gene Selection for Gene Expression Analysis of Oocytes Collected from Dairy Cattle and Buffaloes during Winter and Summer. PLoS One. 2014; 9: e93287.

26. Schroder AL, Pelch KE, Nagel Estrogen modulates expression of putative housekeeping genes in the mouse uterus. Endocrine. 2009; 35: 211-9.

27. Lesage-Padilla A, Forde N, Poirée M, Healey GD, Giraud-Delville C, Reinaud P, Eozenou C, Vitorino Carvalho A, Galio L, Raliou M, Oudin JF, Richard C, Sheldon IM, Charpigny G, Lonergan P, Sandra O. Maternal metabolism affects endometrial expression of oxidative stress and FOXL2 genes in cattle. PLoS One. 2017; 12: e0189942.

28. Van Leeuwen J, Berg DK, Pfeffer PL. Morphological and Gene Expression Changes in Cattle Embryos from Hatched Blastocyst to Early Gastrulation Stages after Transfer of In Vitro Produced Embryos. PLoS One. 2015; 10: e0129787.

29. Vandesompele J, De Preter K, Pattyn F, Poppe B, Van Roy N, De Paepe A, Speleman F. Accurate normalization of real-time quantitative RT-PCR data by geometric averaging of multiple internal control genes. Genome Biol. 2002; 3: research0034.

30. Cieslak J, Mackowski M, Czyzak-Runowska G, Wojtowski J, Puppel K, Kuczynska B, Pawlak P. Screening for the Most Suitable Reference Genes for Gene Expression Studies in Equine Milk Somatic Cells. PLoS One. 2015; 10: e0139688.

31. Saremi B, Sauerwein H, Dänicke S, Mielenz M. Technical note: identification of reference genes for gene expression studies in different bovine tissues focusing on different fat depots. Dairy Sci. 2012; 95: 3131-3138.

32. Ma YF, Wu ZH, Gao M, Loor JJ. Nuclear factor erythroid 2-related factor 2 antioxidant response element pathways protect bovine mammary epithelial cells against $\mathrm{H} 2 \mathrm{O} 2$-induced oxidative damage in vitro. Dairy Sci. 2018; 101: 5329-5344.

33. Cheng X, Yang S, Xu C, Li L, Zhang Y, Guo Y, Zhang C, Li P, Long M, He J. Proanthocyanidins Protect against $\beta$-Hydroxybutyrate-Induced Oxidative Damage in Bovine EndometrialCells. 2019; 24: pii: E400. 
34. Tripurani SK, Wee G, Lee KB, Smith GW, Wang L, Yao J. MicroRNA-212 Post-Transcriptionally Regulates Oocyte-Specific Basic-Helix-Loop-Helix Transcription Factor, Factor in the Germline Alpha (FIGLA), during Bovine Early Embryogenesis. PLoS One. 2013; 8: e76114.

35. Sakumoto R, Hayashi KG, Fujii S, Kanahara H, Hosoe M, Furusawa T, Kizaki K. Possible Rolesof CCand CXC-Chemokines in Regulating Bovine Endometrial Function during Early Pregnancy. J. Mol. Sci. 2017; 18: pii: E742.

36. Denicol AC, Dobbs KB, McLean KM, Carambula SF, Loureiro B, Hansen PJ. Canonical WNT signaling regulates development of bovine embryos to the blastocyst stage. Rep. 2013; 3: 1266.

37. Andersen CL, Jensen JL, Ørntoft TF. Normalization of real-time quantitative reverse transcription-PCR data: A model-based variance estimation approach to identify genes suited for normalization, applied to bladder and colon cancer data sets. Cancer Res. 2004; 64: 5245-5250.

\section{Tables}

Table 1 Primers for selected candidate RGs and target genes. 


\begin{tabular}{|c|c|c|c|c|c|c|}
\hline Genes & $\begin{array}{c}\text { GenBank } \\
\text { accession NO. }\end{array}$ & Primer sequences $\square 5^{\prime}-3^{\prime} \square$ & $\begin{array}{l}\text { Annealing } \\
\text { temperature/ } \\
{ }^{\circ} \mathrm{C}\end{array}$ & $\begin{array}{l}\text { Product } \\
\text { length/bp }\end{array}$ & $\begin{array}{c}\text { Efficiences } \\
(\%)\end{array}$ & $\begin{array}{l}\text { Determination } \\
\text { Coefficient }\end{array}$ \\
\hline \multirow[t]{2}{*}{ SUZ12 } & NM_001205587 & Forward: GAACACCTATCACACACATTCTTGT & 60 & 130 & 92.1 & 0.925 \\
\hline & & Reverse: TAGAGGCGGTTGTGTCCACT & & & & \\
\hline \multirow[t]{2}{*}{ CNOT11 } & XM_002691150.5 & Forward:TCAGTGGACCAAAGCCACCTA & 60 & 169 & 93.8 & 0.996 \\
\hline & & Reverse: CTCCACACCGGTGCTGTTCT & & & & \\
\hline \multirow[t]{2}{*}{ ACTB } & NM_173979.3 & Forward: CGGCATCGAGGACAGGAT & 60 & 169 & 85.3 & 0.917 \\
\hline & & Reverse: CATCGTACTCCTGCTTGCTGAT & & & & \\
\hline \multirow[t]{2}{*}{ RPL19 } & NM_001040516.2 & Forward:TCGCTGTGGCAAGAAGAAAGTCTGG & 60 & 102 & 100.4 & 0.999 \\
\hline & & Reverse: AGCCCATCTTTGATCAGCTTCCG & & & & \\
\hline \multirow[t]{2}{*}{ RPSO } & NM 001101152.2 & Forward: CGAGTATGGGCTCCGGAACAAACG & 60 & 105 & 97.3 & 0.953 \\
\hline & & Reverse: GGGTCTTTCTCATCCAGCGTCAGC & & & & \\
\hline \multirow[t]{2}{*}{ GAPDH } & NM_001034034.2 & Forward:AAGGGCATTCTAGGCTACACTGAGG & 60 & 113 & 94.6 & 0.991 \\
\hline & & Reverse: ACAAAGTGGTCGTTGAGGGCAATGC & & & & \\
\hline \multirow[t]{2}{*}{$T B P$} & NM_001075742.1 & Forward: CCTAAAGACCATTGCACTTCG & 60 & 146 & 85.9 & 0.999 \\
\hline & & Reverse: CTTCACTCTTGGCTCCTGTG & & & & \\
\hline \multirow[t]{2}{*}{ HPRT1 } & NM_001034035.2 & Forward: GGATTACATCAAAGCACTGAACA & 60 & 194 & 94.5 & 0.990 \\
\hline & & Reverse: CATTGTCTTCCCAGTGTCAATT & & & & \\
\hline \multirow[t]{2}{*}{ SDHA } & NM_174178.2 & Forward: GCAGAACCTGATGCTTTGTG & 60 & 185 & 85.0 & 0.960 \\
\hline & & Reverse: CGTAGGAGAGCGTGTGCTT & & & & \\
\hline \multirow[t]{3}{*}{ PPIA } & NM_178320.2 & Forward: & 60 & 120 & 98.3 & 0.989 \\
\hline & & GCAAGCACGTGGTACTTTGGReverse: & & & & \\
\hline & & TTGTCCACAGTCAGCAATGG & & & & \\
\hline NRF2 & NM_001011678.2 & $\begin{array}{l}\text { Forward: CCAGTACCAGCAACGGCATReverse: } \\
\text { TGTTGTGCTTTCAGGGTGGT }\end{array}$ & 60 & 134 & 90.0 & 0.975 \\
\hline \multirow[t]{2}{*}{ RSAD2 } & NM_001045941.1 & Forward: GCTGAAAGAAGCAGGTATGGA & 58 & 176 & 95.0 & 0.969 \\
\hline & & Reverse: CGTACTTCTGGAACCACCTCT & & & & \\
\hline
\end{tabular}

Table 2 Ranking of the stability of the RGs determined by NormFinder. 


\begin{tabular}{ccc}
\hline Gene & stability & Rank \\
\hline PPIA & 0.394 & 1 \\
CNOT11 & 0.422 & 2 \\
RPL19 & 0.453 & 3 \\
RPS9 & 0.453 & 4 \\
TBP & 0.476 & 5 \\
ACTB & 0.495 & 6 \\
SUZ12 & 0.575 & 7 \\
SDHA & 0.577 & 8 \\
HPRT1 & 0.597 & 9 \\
GAPDH & 0.687 & 10 \\
CNOT11+PPIA & 0.264 & Best combination \\
\hline
\end{tabular}

Table 3 Ranking of the stability of the RGs determined by Bestkeeper.

\begin{tabular}{|c|c|c|c|c|c|}
\hline Gene & Standard deviation (SD) & coefficient of variance (CV) & Correlation coefficient $(r)$ & P-value & Rank \\
\hline RPS9 & 1.00 & 4.67 & 0.834 & 0.001 & 1 \\
\hline CNOT11 & 0.81 & 3.05 & 0.831 & 0.001 & 2 \\
\hline$T B P$ & 0.90 & 3.26 & 0.807 & 0.001 & 3 \\
\hline$R P L 19$ & 0.79 & 3.82 & 0.773 & 0.001 & 4 \\
\hline SUZ12 & 0.71 & 2.70 & 0.567 & 0.004 & 5 \\
\hline
\end{tabular}

Table 4 Ranking of the stability of the RGs determined by the three programs.

\begin{tabular}{ccccc}
\hline Gene & \multicolumn{3}{c}{ Programs } & Average Rank \\
\cline { 2 - 4 } & GeNorm & NormFinder & Bestkeeper & \\
\hline SUZ12 & 8 & 7 & 5 & 6.67 \\
CNOT11 & 3 & 2 & 2 & 2.33 \\
RPL19 & 1 & 3 & 4 & 2.67 \\
RPS9 & 2 & 4 & 1 & 2.33 \\
TBP & 6 & 5 & 3 & 4.67 \\
\hline
\end{tabular}



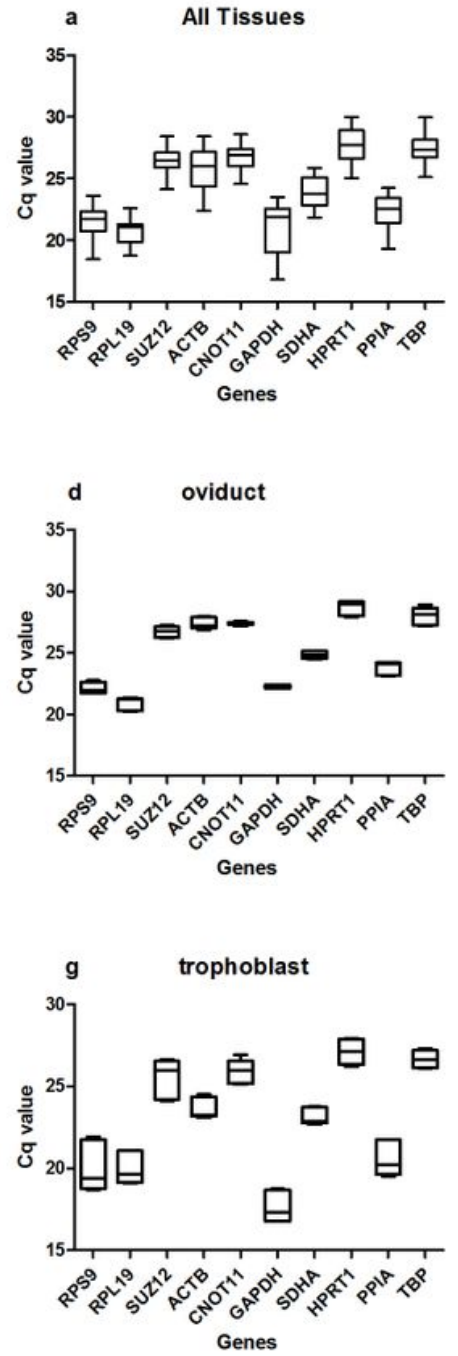
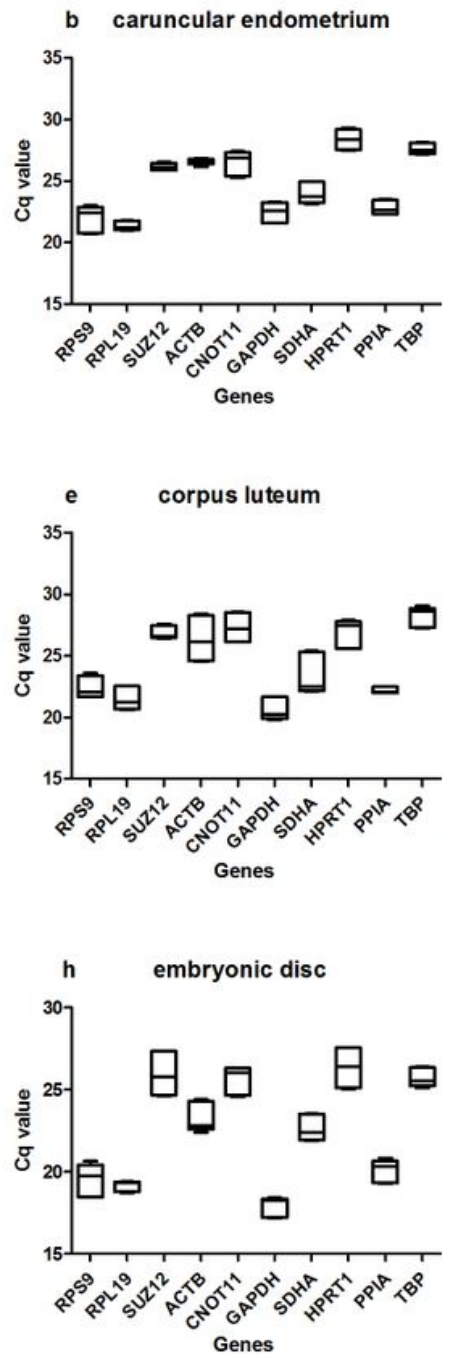
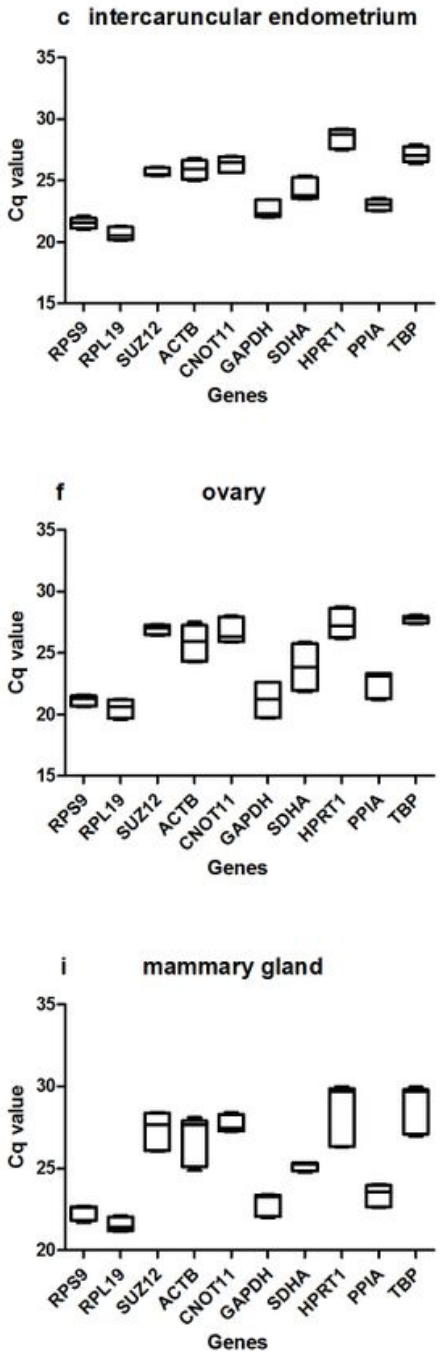

Figure 1

Box plot of $\mathrm{Cq}$ values. The variations in the quantification cycle $(\mathrm{Cq})$ values are shown for all detected genes from maternal and fetal tissues of pregnant cows. Each box represents $50 \%$ of the most frequent Cq value. Whiskers depict the $25 \%$ minimum and maximum Cq values. The median is represented by the line in each box. (a), all tissues. (b), caruncular endometrium. (c), intercaruncular endometrium. (d), oviduct. (e), corpus luteum. (f), ovary. (g), trophoblast. (h), embryonic disc. (i), mammary gland. 

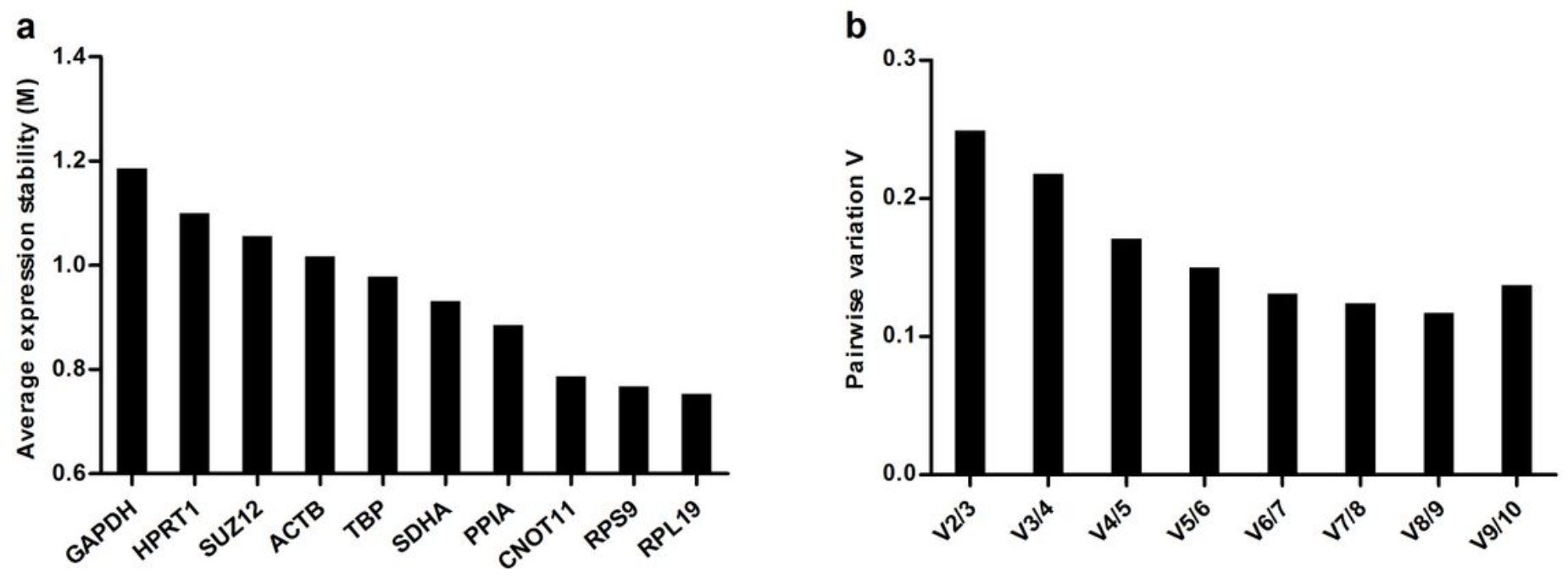

Figure 2

GeNorm calculations. (a) Stability of reference gene expression (M) calculated in GeNorm software. Low $M$ values indicate the best RGs, and high $M$ values indicate the worst RGs. (b) Determination of the optimal number of RGs for normalization. The GeNorm V value shows the pairwise variation between the normalization factors of $R G s(V n / V n+1)$, and the optimal number of $R G s$ is indicated by values of GeNorm V below 0.15 . 

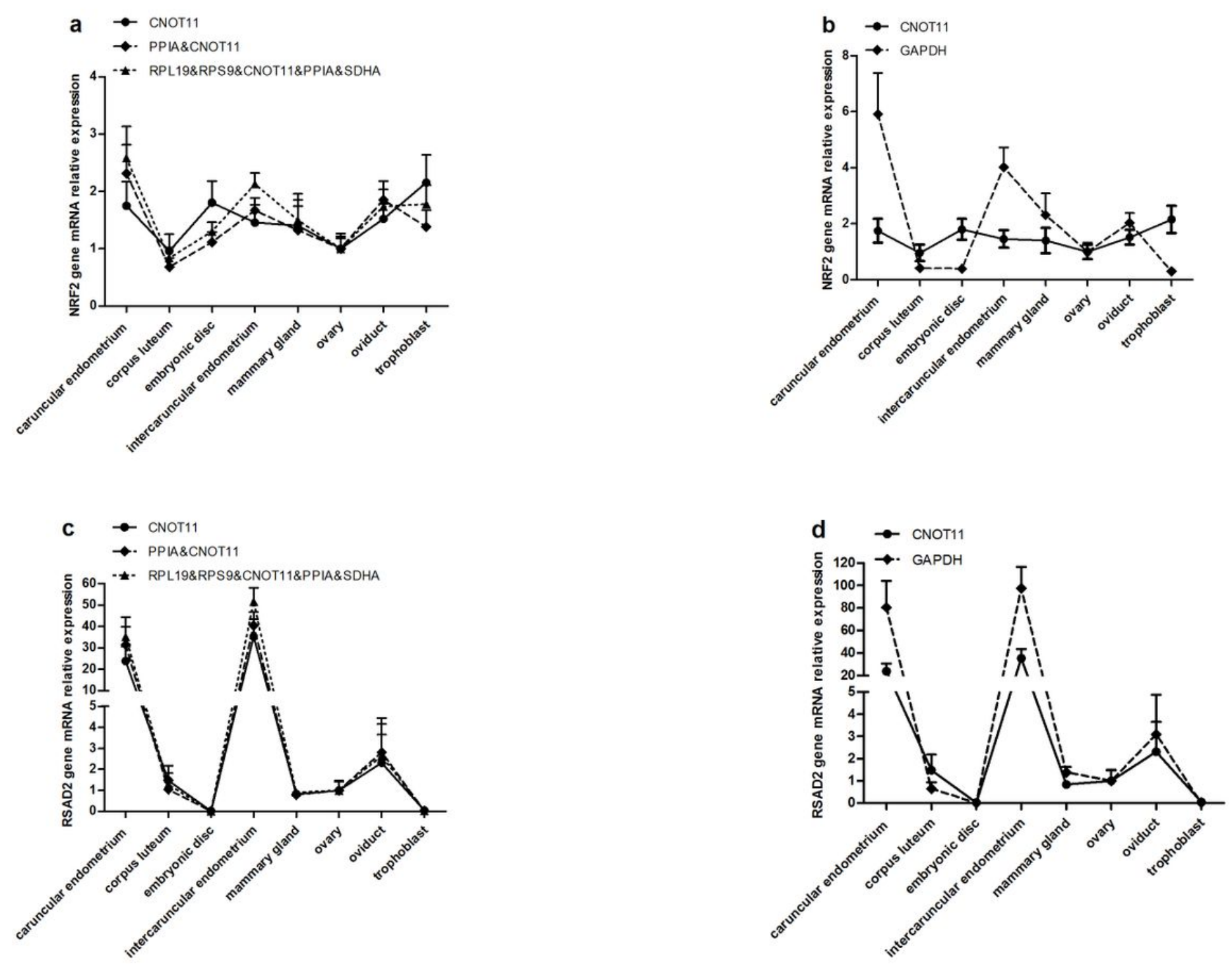

Figure 3

Relative gene expression results for NRF2 and RSAD2 after normalization to reference genes with different stability in maternal and fetal tissues of pregnant dairy cows (in contrast to ovary tissue). (a), (c) The relative gene expression of NRF2 or RSAD2 gene after normalization to the most stable gene (CNOT11) identified by the three programs, the optimum pair (CNOT11 and PPIA) of RGs identified by NormFinder, and the five best genes according to GeNorm (SDHA, PPIA, CNOT11, RPS9 and RPL19). (b), (d) The relative gene expression of NRF2 and RSAD2 after normalization to the most stable gene (CNOT11) and the least stable gene (GAPDH) identified by the three programs.

\section{Supplementary Files}

This is a list of supplementary files associated with this preprint. Click to download.

- TableS1andS2.doc 
- Fig.S1.jpg

Page 20/20 